\title{
Linear groups saturated by subgroups of finite central dimension
}

\author{
N. N. Semko, L. V. Skaskiv, and O. A. Yarovaya
}

Communicated by I. Ya. Subbotin

\section{Dedicated to Leonid Kurdachenko for his 70th birthday}

Abstract. Let $F$ be a field, $A$ be a vector space over $F$ and $G$ be a subgroup of $\operatorname{GL}(F, A)$. We say that $G$ has a dense family of subgroups, having finite central dimension, if for every pair of subgroups $H, K$ of $G$ such that $H \leqslant K$ and $H$ is not maximal in $K$ there exists a subgroup $L$ of finite central dimension such that $H \leqslant L \leqslant K$. In this paper we study some locally soluble linear groups with a dense family of subgroups, having finite central dimension.

\section{Introduction}

We recall that a group $G$ that is isomorphic to a group of automorphisms of a vector space $A$ over a field $F$ is called a linear group and we denote the group of all such automorphisms by $\operatorname{GL}(F, A)$. If $\operatorname{dim}_{F}(A)$, the dimension of $A$ over $F$, is finite, say $n$, then we say that $G$ is a finite dimensional linear group and it is then well-known that $\mathrm{GL}(F, A)$ can be identified with the group of $n \times n$ matrices with entries in $F$. From the outset, finite dimensional linear groups have played an important role in group theory. This is partly due to the correspondence mentioned above, but also because of the rich interplay between geometrical and algebraic ideas associated with such groups.

2010 MSC: Primary 20E15, 20F16; Secondary 20E25, 20E34, 20F22, 20 F50.

Key words and phrases: linear group, infinite group, infinite dimensional linear group, dense family of subgroups, locally soluble group, finite central dimension. 
The study of the subgroups of $\mathrm{GL}(F, A)$ in the case when $A$ is infinite dimensional over $F$ has been much more limited and normally requires some additional restrictions. There is quite a large array of papers that show the effectiveness of applying various natural restrictions for the study of infinite dimensional linear groups (see survey articles [1-4]). One area that proved to be quite effective was the study of linear groups that has a very big family of subgroups, having finite central dimension.

Let $G$ be a subgroup of $\operatorname{GL}(F, A)$ and $Z=C_{A}(G)$. It is not hard to see that a subspace $Z$ is $G$-invariant and $G$ acts trivially on $Z$. Therefore, we see that $G$ actually acts on the quotient-space $A / Z$.

Let $G$ be a subgroup of $\operatorname{GL}(F, A)$. Then the central dimension of $G$ is a dimension of the quotient-space $A / \zeta_{G}(A)$. In particular, if $\operatorname{dim}_{F}\left(A / \zeta_{G}(A)\right)$ is finite, then we will say that $G$ has finite central dimension. According to the definition, linear groups, having finite central dimension, quite close to finite dimensional linear groups. Therefore, it was obvious to study the infinite dimensional linear groups, saturated by subgroups of finite central dimension. Among the works on this subject, we can highlight $[1,5-10]$.

Among the restrictions that contributed a significant role in the study of both finite and infinite groups, we can highlight the restriction associated with the presence of one or another family of dense subgroups in a group. Let $\mathcal{P}$ be a some property. We say that a group $G$ has a dense family of subgroups, having property $\mathcal{P}$, if for every pair of subgroups $H, K$ of $G$ such that $H \leqslant K$ and $H$ is not maximal in $K$ there exists a subgroup $L$, having property $\mathcal{P}$, such that $H \leqslant L \leqslant K$ (we can note that $L$ can match with one of the subgroups $H$ or $K$ ).

Groups with different natural dense family have been considered by many authors (see, for example, [11-20]).

In this paper we will apply this restriction for a study of infinite dimensional linear groups.

Let $F$ be a field, $A$ be a vector space over $F$ and $G$ be a subgroup of $\operatorname{GL}(F, A)$. We say that $G$ has a dense family of subgroups, having finite central dimension, if for every pair of subgroups $H, K$ of $G$ such that $H \leqslant K$ and $H$ is not maximal in $K$ there exists a subgroup $L$ of finite central dimension such that $H \leqslant L \leqslant K$ (we can note that $L$ can match with one of the subgroups $H$ or $K$ ).

Note that infinite dimensional linear groups, whose proper subgroups have finite central dimension, has this property. Locally soluble groups, whose proper subgroups have finite central dimension, were studied in [5]. 
Therefore, in this paper, the study of linear groups with a dense family of subgroups, having finite central dimension, will be conducted under the additional condition of their local solvability.

The main result of this paper is the following

Theorem 1. Let $F$ be a field, $A$ be a vector space over $F$, having infinite dimension, and $G$ be a locally soluble subgroup of $\mathrm{GL}(F, A)$. Suppose that $G$ has infinite central dimension. If $G$ has a dense family of subgroups having finite central dimension, then $G$ is a group of one of following types:

(i) $G$ is cyclic or quasicyclic p-group for some prime $p$;

(ii) $G=K \times L$ where $K$ is cyclic or quasicyclic p-group for some prime $p$ and $L$ is a group of prime order;

(iii) $G=\left\langle a, b|| a\left|=2^{n},\right| b\right|=2, a^{b}=a^{t}$ where $\left.t=1+2^{n-1}, n \geqslant 3\right\rangle$;

(iv) $G=\left\langle a, b|| a\left|=2^{n},\right| b\right|=2, a^{b}=a^{t}$ where $\left.t=-1+2^{n-1}, n \geqslant 3\right\rangle$;

(v) $G=\left\langle a, b|| a\left|=2^{n},\right| b \mid=2, a^{b}=a^{-1}\right\rangle$;

(vi) $G=\langle a, b|| a|=2^{n}, b^{2}=a^{t}$ where $\left.t=2^{n-1}, a^{b}=a^{-1}\right\rangle$;

(vii) $G=\left\langle a, b|| a\left|=p^{n},\right| b \mid=p, a^{b}=a^{t}, t=1+p^{n-1}, n \geqslant 2\right\rangle, p$ is an odd prime;

(viii) $G=\langle a\rangle \lambda\langle b\rangle,|a|=p^{n}$ where $p$ is an odd prime, $|b|=q$, $q$ is a prime, $q \neq p$

(ix) $G=B \lambda\langle a\rangle,|a|=p^{n}, B=C_{G}(B)$ is an elementary abelian $q$ subgroup, $p$ and $q$ are primes, $p \neq q, B$ is a minimal normal subgroup of $G$;

(x) $G=K \lambda\langle b\rangle$, where $K$ is a quasicyclic 2-subgroup, $|b|=2$ and $x^{b}=x^{-1}$ for each element $x \in K$;

(xi) $G=K\langle b\rangle$, where $K=\left\langle a_{n} \mid a_{1}^{p}=1, a_{n+1}^{p}=a_{n}, n \in \mathbb{N}\right\rangle$ is a quasicyclic 2-subgroup, $b^{2}=a_{1}$ and $a_{n}^{b}=a_{n}^{-1}, n \geqslant 2$;

(xii) $G=K \lambda\langle b\rangle$, where $K$ is a quasicyclic $p$-subgroup, $p$ is an odd prime, $K=C_{G}(K),|b|=q$ is a prime such that $p \neq q$;

(xiii) $G=Q \lambda K$, where $K$ is a quasicyclic p-subgroup, $Q=C_{G}(Q)$ is an elementary abelian $q$-subgroup, $p, q$ are primes, $p \neq q, Q$ is a minimal normal subgroup of $G$.

\section{Preliminary results}

Lemma 1. Let $F$ be a field, $A$ be a vector space over $F$ and $G$ be a subgroup of $\operatorname{GL}(F, A)$.

(i) If $H, K$ are two subgroups of $G$ such that $H \leqslant K$ and $K$ has finite central dimension, then $H$ has finite central dimension. 
(ii) If $H, K$ are two subgroups of $G$, having finite central dimension, then the both subgroups $\langle H, K\rangle$ and $H \cap K$ have finite central dimension.

(iii) If $G$ has finite central dimension and $\operatorname{char}(F)=p$ is a prime, then $G$ includes a normal elementary abelian p-subgroup $L$ such that $G / L$ is isomorphic to some subgroup of a group $\mathrm{GL}(n, F)$ where $n=\operatorname{dim}_{F}\left(A / C_{A}(G)\right)$.

(iv) If $G$ has finite central dimension and $\operatorname{char}(F)=0$, then $G$ includes a normal abelian torsion-free subgroup $L$ such that $G / L$ is isomorphic to some subgroup of $\mathrm{GL}(n, F)$ where $n=\operatorname{dim}_{F}\left(A / C_{A}(G)\right)$.

Proof. (i) If $K$ has finite central dimension, then $C_{A}(K)$ has finite codimension in a vector space $A$. An inclusion $H \leqslant K$ implies that $C_{A}(K) \leqslant$ $C_{A}(H)$. It follows that $C_{A}(H)$ has finite codimension in $A$.

(ii) Clearly $C_{A}(\langle H, K\rangle)$ and $C_{A}(H \cap K)$ includes $C_{A}(H) \cap C_{A}(K)$. Note that the last subspace has finite codimension in $A$. It follows that $\operatorname{codim}_{F}\left(C_{A}(\langle H, K\rangle)\right)$ and $\operatorname{codim}_{F}\left(C_{A}(H \cap K)\right)$ are finite.

(iii)-(iv) Let $L=C_{G}\left(A / C_{A}(G)\right)$, then $G / L$ is isomorphic to a subgroup of $\mathrm{GL}\left(F, A / C_{A}(G)\right) \cong \mathrm{GL}(n, F)$ where $n=\operatorname{centdim}_{F}(G)$. If $x \in L$, then $x(a)=a+c$ for some element $c \in C_{A}(G)$. In other words, $x(a)-a=$ $(x-1)(a) \in C_{A}(G)$ for every element $a \in A$. If $y$ is another element of $L$, then

$$
(x y)(a)=x(y(a))=x(a+(y-1)(a))=a+(x-1)(a)+(y-1)(a) .
$$

In a similar way we obtain that $(y x)(a)=a+(y-1)(a)+(x-1)(a)$. Since it is true for every element $a$ of $A, x y=y x$. If $L$ is non-trivial, then for every element $1 \neq x \in L$ there is an element $d \in A$ such that $x(d) \neq d$. It follows that $d_{1}=(x-1)(d) \neq 0$. We have

$$
x^{2}(d)=x(x(d))=x\left(d+d_{1}\right)=x(d)+d_{1}=d+d_{1}+d_{1}=d+2 d_{1} .
$$

Using an ordinary induction, we obtain that $x^{n}(d)=d+n d_{1}$. Thus we can see that if $\operatorname{char}(F)=p$ is a prime, then $x^{p}=1$. If $\operatorname{char}(F)=0$, then $n d_{1} \neq 0$ for all positive integer $n$, so that $x^{n} \neq 1$ for all positive integer $n$. This means that $L$ is a torsion-free subgroup.

Corollary 1. Let $F$ be a field, $A$ be a vector space over $F$, having infinite dimension, and $G$ be a subgroup of $\mathrm{GL}(F, A)$. If $H, K$ are two subgroups of $G$ such that $H \leqslant K$ and a subgroup $H$ has infinite central dimension, then $K$ has infinite central dimension. 
Corollary 2. Let $F$ be a field, $A$ be a vector space over $F$, having infinite dimension, and $G$ be a subgroup of $\operatorname{GL}(F, A)$. If $G$ has a dense family of subgroups having finite central dimension, then every subgroup of $G$, having infinite central dimension, is maximal.

Lemma 2. Let $F$ be a field, $A$ be a vector space over $F$, having infinite dimension, and $G$ be a subgroup of $\mathrm{GL}(F, A)$. Suppose that $G$ includes the subgroups $B, K$ satisfying the following conditions:

(i) $B$ is an infinite elementary abelian p-subgroup and $K$ is a quasicyclic p-subgroup for some prime $p$;

(ii) $B$ is $K$-invariant;

(iii) $B \cap K=\langle 1\rangle$.

If $G$ has a dense family of subgroups, having finite central dimension, then $K$ has finite central dimension.

Proof. Being a soluble $p$-subgroup, $B K$ is locally nilpotent. Since $B$ is infinite, it includes a proper non-trivial $K$-invariant subgroup (see, for example, [21, Proposition 1.2.20]). It follows that $K$ is a not maximal subgroup of $B K$. In this case there exists a subgroup $L$, having infinite central dimension, such that $K \leqslant L \leqslant B K$. Using Lemma 1 we obtain that $K$ has finite central dimension.

Corollary 3. Let $F$ be a field, $A$ be a vector space over $F$, having infinite dimension, and $G$ be a subgroup of $\mathrm{GL}(F, A)$. Suppose that $G$ includes the subgroups $B, K$ satisfying the following conditions:

(i) $B$ is an infinite elementary abelian p-subgroup and $K$ is a quasicyclic p-subgroup for some prime $p$;

(ii) $B$ is $K$-invariant;

(iii) $B \cap K$ is finite.

If $G$ has a dense family of subgroups, having finite central dimension, then $K$ has finite central dimension.

Proof. Indeed, a subgroup $B \cap K$ is $K$-invariant and $B /(B \cap K)$ is infinite, so that an intersection $B /(B \cap K) \cap K /(B \cap K)$ is trivial, and we can apply Lemma 2 .

Lemma 3. Let $F$ be a field, $A$ be a vector space over $F$, having infinite dimension, and $G$ be a subgroup of $\mathrm{GL}(F, A)$. Suppose that $G$ includes the subgroups $B, K$ satisfying the following conditions:

(i) $B$ is an infinite elementary abelian p-subgroup and $K$ is a quasicyclic $q$-subgroup where $p, q$ are primes and $p \neq q$; 
(ii) $B$ is $K$-invariant.

If $G$ has a dense family of subgroups, having finite central dimension, then either $K$ has finite central dimension or $B$ is a minimal $K$-invariant subgroup.

Proof. Suppose that $K$ has infinite central dimension. If we suppose that $B$ includes a proper non-trivial $K$-invariant subgroup $C$, then a subgroup $K$ is not maximal in $B K$. In this case there exists a subgroup $L$, having infinite central dimension, such that $K \leqslant L \leqslant B K$. Using Lemma 1 we obtain that $K$ has finite central dimension. This contradiction shows that $B$ is a minimal $K$-invariant subgroup.

Lemma 4. Let $F$ be a field, $A$ be a vector space over $F$, having infinite dimension, and $G$ be a locally soluble subgroup of $\mathrm{GL}(F, A)$. Suppose that $G$ includes a subgroup $K$, having infinite central dimension. If $G$ has a dense family of subgroups, having finite central dimension, then $K$ satisfies the following conditions:

(i) $K$ is quasicyclic or cyclic $p$-subgroup for some prime $p$;

(ii) every proper subgroup of $K$ has finite central dimension;

(iii) $K$ is a maximal subgroup of $G$.

Proof. Corollary 2 shows that $K$ is a maximal subgroup of $G$. Hence if we suppose that $K$ includes a proper subgroup $H$, having infinite central dimension, then from Corollary 2 we obtain that a subgroup of $H$ must be maximal in $G$, what is impossible. This contradiction shows that every proper subgroup of $K$ has finite central dimension. If $K$ is infinite, then $K$ is a quasicyclic $p$-subgroup for some prime $p$ [5, Corollaries 5.4, 5.6]. Therefore consider a case when $K$ is finite. Being finite, $K$ is soluble. Then $D=[K, K] \neq K$. If we suppose that a set $\Pi(K / D)$ contains two different primes, then $K$ is a product of two proper normal subgroups. In this case $K$ has finite central dimension, as shows Lemma 1. Thus $K / D$ is a $p$-group for some prime $p$. If we suppose that $K / D$ is not cyclic, then again $K$ is a product of two proper normal subgroups, which follows that $K$ has finite central dimension. This contradiction shows that $K / D$ is a cyclic $p$-subgroup. Let $x$ be an element of $K$ such that $K / D=\langle x D\rangle$. Without loss of generality we may assume that $x$ is a $p$-element. If we suppose that $\langle x\rangle$ is a proper subgroup of $K$, then an equality $K=\langle x\rangle D$ together with Lemma 1 imply, that $K$ has finite central dimension. This contradiction proves that $K=\langle x\rangle$. 


\section{Proof of the main theorem}

If $G$ is infinite and every proper subgroup of $G$ has finite central dimension, then $G$ is a quasicyclic $p$-subgroup for some prime $p$ [5, Corollaries 5.4,5.6]. If $G$ is finite and every proper subgroup of $G$ has finite central dimension, then using the arguments from a proof of Lemma 4, we obtain that $G$ is a cyclic $p$-subgroup for some prime $p$. In this case $G$ is a group of type (i).

Suppose now that $G$ includes a proper subgroup $K$, having infinite central dimension. Then Lemma 4 shows that $K$ is a maximal subgroup of $G$, every proper subgroup of $K$ has finite central dimension and $K$ is a cyclic or quasicyclic $p$-subgroup for some prime $p$.

Consider first a case when $K=\langle a\rangle$ is a cyclic $p$-subgroup. Choose an element $x$ such that $x \notin\langle a\rangle$. The fact that $\langle a\rangle$ is a maximal subgroup of $G$ implies that $G=\langle a, x\rangle$. In particular, $G$ is soluble. If $a x=x a$, then $G$ is abelian. In this case it is not hard to prove that $G$ is a group of type (ii). Therefore suppose that $x a \neq a x$. In this case $G$ is non-abelian. Being soluble, $G$ has a finite series of normal subgroups

$$
\langle 1\rangle=S_{0} \leqslant S_{1} \leqslant \ldots \leqslant S_{n}=G
$$

whose factors are periodic abelian or torsion-free abelian, includes a normal abelian subgroup $U$. There exists a number $k$ such that $S_{k} \leqslant\langle a\rangle$ but $\langle a\rangle$ does not include $S_{k+1}$. Then $G=\left\langle a, S_{k+1}\right\rangle$. If $S_{k+1} / S_{k}$ is periodic, then $S_{k+1}$ is periodic and therefore $G$ is periodic. Being finitely generated and soluble, $G$ is finite. Assume that $S_{k+1} / S_{k}$ is torsion-free abelian. An equality $G=\left\langle a, S_{k+1}\right\rangle$ implies that $S_{k+1} / S_{k}$ is finitely generated. Then $\left(S_{k+1} / S_{k}\right)^{q}=U / S_{k}$ for every prime $q$, so that $\langle a\rangle \neq\langle a, U\rangle \neq\left\langle a, S_{k+1}\right\rangle$, and we obtain a contradiction with the maximality of $\langle a\rangle$. This contradiction shows that $G$ is finite. Consider a case when a subgroup $\langle a\rangle$ is normal in $G$. Then $|G /\langle a\rangle|=q$ is a prime. If $q=p$, then $G$ is a $p$-group, having a maximal cyclic subgroup. The structure of such groups was described (see, for example, [22, §1, Theorem 1.2]), so we come to the groups of types (iii)-(vii).

Suppose that a subgroup $\langle a\rangle$ is not normal in $G$. Being finite and soluble, $G$ has a finite series of normal subgroups

$$
\langle 1\rangle=S_{0} \leqslant S_{1} \leqslant \ldots \leqslant S_{n}=G
$$

whose factors are elementary abelian and $G$-chief. There exists a number $k$ such that $S_{k} \leqslant\langle a\rangle$ but $\langle a\rangle$ does not include $S_{k+1}$. Then $G=\left\langle a, S_{k+1}\right\rangle$. 
If $S_{k+1} / S_{k}$ is a $p$-group, then $G$ is also $p$-group. But in finite $p$-group each maximal subgroup is normal, this case has been considered above. Thus $S_{k+1} / S_{k}$ is an elementary abelian $q$-group, where $q \neq p$. Since $\langle a\rangle$ is a maximal subgroup, then either $N_{G}(\langle a\rangle)=\langle a\rangle$ or $N_{G}(\langle a\rangle)=G$. On the other hand, $\langle a\rangle$ is not normal, therefore a second case is impossible. An equality $N_{G}(\langle a\rangle)=\langle a\rangle$ implies that $G=B \lambda\langle a\rangle$, where $B$ is a normal Sylow $p^{\prime}$-subgroup of $G$ (see, for example, [23, Chapter 7, Theorem 4.3]), $\langle a\rangle$ is a $p$-group. Together with an equality $G=\left\langle a, S_{k+1}\right\rangle$ and the fact that $S_{k+1} / S_{k}$ is a $G$-chief elementary abelian $q$-factor it follows that $B$ is an elementary abelian $q$-subgroup and $B$ is a minimal normal subgroup of $G$. It shows that $G$ is a group of type (ix).

Consider now a case when $K$ is a quasicyclic $p$-subgroup. Lemma 4 shows that $K$ is a maximal subgroup of $G$. If $C_{G}(K) \neq K$, then $C_{G}(K)=$ $G$. In this case $K \leqslant \zeta(G)$, in particular, $K$ is normal in $G$. Then $G / K$ has prime order. In turn out, it follows that $G$ is abelian. Since $K$ is divisible, $G=K \times L$ (see, for example, [24, Theorem 21.2]), where $L \cong G / K$ is a group of prime order. Thus $G$ is a group of type (ii).

Further we will suppose that $C_{G}(K)=K$. For a subgroup $N_{G}(K)$ we have also two possibility: $N_{G}(K)=G$ or $N_{G}(K)=K$. Consider a first case. Then $K$ is maximal and normal in $G$, so that $G / K$ is a group of prime order. An equality $C_{G}(K)=K$ shows that $G / K$ is isomorphic to a subgroup of $\operatorname{Aut}(K)$. We have $\operatorname{Aut}(K)=C \times J$ where $|C|=2$ and $J$ is an additive group of 2-adic integer, whenever $p=2$, or $\operatorname{Aut}(K)=C \times J$ where $C$ is a cyclic group of order $p-1$ and $J$ is an additive group of $p$-adic integer, whenever $p$ is an odd prime (see, for example, [25, Chapter 4, Theorem 6.5]). Thus either $|G / K|=2$ whenever $p=2$, or $|G / K|=q$ is a prime, $q \neq p$ whenever $p$ is an odd prime.

Suppose that $G$ is a 2-group and $G / K$ is a group of order 2. If $G$ contains an element $b$ of order 2 such that $b \notin K$, then $G=K \lambda\langle b\rangle$ and $x^{b}=x^{-1}$ for every element $x \in K$. In other words, $G$ is a group of type (x). Suppose now that for every element $c \notin K$ the intersection $\langle c\rangle \cap K$ is non-trivial. We have

$$
K=\left\langle a_{n} \mid a_{1}^{p}=1, a_{n+1}^{p}=a_{n}, n \in \mathbb{N}\right\rangle .
$$

Let $n \geqslant 2$ and consider a subgroup $\left\langle a_{n}, c\right\rangle$. Then $\left\langle a_{n}, c\right\rangle$ has a cyclic subgroup of index 2 . From the description of the structure of finite $p$-groups, having a maximal cyclic subgroup (see, for example, [22, §1, Theorem 1.2]), we obtain that $\left\langle a_{n}, c\right\rangle$ has an element $b$ such that $b^{2}=a_{1}$ and $a_{n}^{b}=a_{n}^{-1}$. 
Thus we obtain that $G=K\langle b\rangle$, where $b^{2}=a_{1}$ and $a_{n}^{b}=a_{n}^{-1}$ for all $n \geqslant 2$. Thus $G$ is a group of type (xi).

Suppose now that $K$ is a $p$-subgroup where $p>2$. As we have seen above, in this case $G / K$ is a group of prime order $q$ and $q \neq p$. Then $G=K \lambda\langle b\rangle$ (see, for example, [26, Theorem 2.4.5]), where $b$ is an element of order $q$, so that $G$ is a group of type (xii).

Suppose now that a subgroup $K$ is not normal in $G$. Moreover, without loss of generality we may suppose that $G$ does not include proper normal subgroups, having infinite dimension (this case was considered above). Since $G$ is infinite and non-abelian, $G$ includes a non-trivial proper normal subgroup $H$ (see, for example, [21, Proposition 1.2.18]). Suppose that $K$ does not include $H$, then $G=H K$. The fact that a subgroup $H$ has finite central dimension means that $C_{A}(H)$ has finite dimension over $F$. The factor-group $H / C_{H}\left(A / C_{A}(H)\right)$ is isomorphic to some subgroup of $\operatorname{GL}(n, F)$ where $n=\operatorname{dim}_{F}\left(A / C_{A}(H)\right)$. Being locally soluble, $H / C_{H}\left(A / C_{A}(H)\right)$ is soluble (see, for example, [27, Corollary 3.8]). As we can see from the proof of Lemma $1, C_{H}\left(A / C_{A}(H)\right)$ is an abelian subgroup. It follows that $H$ is also soluble. An equality $G=H K$ and the fact that $K$ is abelian imply that $G$ is soluble. Then $G$ has a finite series of normal subgroups

$$
\langle 1\rangle=S_{0} \leqslant S_{1} \leqslant \ldots \leqslant S_{n}=G
$$

whose factors are abelian and $G$-chief. Moreover, we can assume that each factor $S_{j+1} / S_{j}$ is either periodic or torsion-free. There exists a number $k$ such that $S_{k} \leqslant K$ but $K$ does not include $S_{k+1}$. Then $G=K S_{k+1}$.

Suppose first that $S_{k+1} / S_{k}$ is periodic. If we assume that $\Pi\left(S_{k+1} / S_{k}\right)$ contains at least two primes, then $S_{k+1} / S_{k}=X_{1} / S_{k} \times X_{2} / S_{k}$ where the both subgroups $X_{1}, X_{2}$ are $G$-invariant, $S_{k} \neq X_{1} \neq S_{k+1}, S_{k} \neq X_{2} \neq S_{k+1}$. But in this case $K$ is not maximal in $G$. This contradiction proves that $S_{k+1} / S_{k}$ is a $q$-group for some prime $q$. If we suppose that $\Omega_{1}\left(S_{k+1} / S_{k}\right) \neq$ $S_{k+1} / S_{k}$, then we again obtain a contradiction with a maximality of $K$. It shows that $S_{k+1} / S_{k}$ is an elementary abelian $q$-group. If $q=p$, then $G$ is a soluble $p$-group. In particular, $G$ is locally nilpotent. But in a locally nilpotent group each maximal subgroup is normal (see, for example, [28, Theorem 18.1.3]), so that $K$ is normal. This contradiction shows that $q \neq p$. An inclusion $S_{k} \leqslant K$ shows that in this case $S_{k}$ is a normal finite Sylow $p$-subgroup of $S_{k+1}$. Then $S_{k+1}=S_{k} \lambda Q$ and every complement to $S_{k}$ in $S_{k+1}$ is conjugated with $Q$ (see, for example, [26, Theorem 2.4.5]), where $Q$ is a Sylow $q$-subgroup of $S_{k+1}$. An equality $G=K S_{k+1}$ proves that $Q$ is a Sylow $q$-subgroup of $G$. An isomorphism 
$Q \cong S_{k+1} / S_{k}$ shows that $Q$ is an elementary abelian $q$-subgroup. Since $G / S_{k+1}$ is a $p$-group, $S_{k+1}$ includes an arbitrary Sylow $q$-subgroup $Q_{1}$ of $G$. Being a maximal $q$-subgroup of $G, Q_{1}$ is a Sylow $q$-subgroup of $S_{k+1}$. An equality $S_{k+1}=Q S_{k}$ shows that $Q$ has finite index in $S_{k+1}$. Then $Q$ includes a normal in $S_{k+1}$ subgroup $R$, having finite index in $S_{k+1}$. An inclusion $Q_{1} \leqslant S_{k+1}$ implies that $R Q_{1}$ is a $q$-subgroup. Then $R Q_{1}=Q_{1}$, that is $R \leqslant Q_{1}$. Since $S_{k+1} / R$ if finite, the set of all Sylow $q$-subgroups of $G$ is finite. In this case all Sylow $q$-subgroup of $G$ are conjugate (see, for example, [26, Lemma 2.3.2]). Then $N_{G}(Q)$ has finite index in $G$, so that index $\left|K: N_{K}(Q)\right|$ is finite. On the other hand, $K$ does not include proper subgroups of finite index. It follows that $K=N_{K}(Q)$. An equality $G=K S_{k+1}=K\left(S_{k} Q\right)=K Q$ implies that $Q$ is a normal subgroup of $G$. Lemma 3 shows that $Q$ is a minimal normal subgroup of $G$, so that $G$ is a group of type (xiii).

Suppose now that $S_{k+1} / S_{k}$ is torsion-free. Let $d \in S_{k+1} \backslash S_{k}, D / S_{k}=$ $\langle d\rangle^{G} S_{k}$. Then $D / S_{k}$ is a normal torsion-free abelian subgroup. The fact that $K$ is a maximal subgroup of $G$ implies that $D K=G$. There exists a prime $r$ such that $D_{1} / S_{k}=\left(D / S_{k}\right)^{r} \neq D / S_{k}$ [29, Theorem 1.15]. But then $K \neq D_{1} K \neq D K=G$, and we obtain a contradiction with a maximality of $K$. This contradiction shows that $S_{k+1} / S_{k}$ can not be torsion-free.

Finally, suppose that $K$ includes every proper normal subgroup of $G$. Then the fact that $K$ is not normal in $G$ implies that $G$ has a finite maximal normal subgroup $M$. Being the chief factor of locally soluble group, $G / M$ is abelian, moreover, it is either elementary abelian or torsionfree abelian (see, for example, [21, Proposition 1.2.18]). But then $K / M$ is normal in $G$, and we obtain a contradiction. This finally contradiction proves a result.

\section{References}

[1] M.R. Dixon, L.A. Kurdachenko, Linear groups with infinite central dimension, Lond. Math. S., 339, 2007, pp. 306-312.

[2] M.R. Dixon, L.A. Kurdachenko, Abstract and linear groups with some specific restrictions, Proceedings of the "Meeting on Group Theory and its applications, on the occasion of Javier Otal's 60th birthday" (Zaragoza, June 10-11, 2011), Madrid, 2012, pp. 87-106.

[3] M.R. Dixon, L.A. Kurdachenko, J.M. Muños-Escolano, J. Otal, Trends in infinite dimensional linear groups, Lond. Math. S., 387, 2011, pp. 271-282.

[4] L.A. Kurdachenko, On some infinite dimensional linear groups, Note Mat., 30(1), 2010, pp. 21-36. 
[5] M.R. Dixon, M.J. Evans, L.A. Kurdachenko, Linear groups with the minimal condition on subgroups of infinite central dimension, J. Algebra, 277(1), 2004, pp. $172-186$.

[6] L.A. Kurdachenko, J. Otal, I. Ya. Subbotin, Linear groups with the maximal condition on subgroups of infinite central dimension, Publ. Mat., 50(1), 2006, pp. 103-131.

[7] L.A. Kurdachenko, J.M. Muños-Escolano, J. Otal, Antifinitary linear groups, Forum Math., 20(1), 2008, pp. 27-44.

[8] L.A. Kurdachenko, J.M. Muños-Escolano, J. Otal, Soluble linear groups with some restrictions on subgroups of infinite central dimension, Ischia Group Theory 2008, 2009, pp.156-173.

[9] L.A. Kurdachenko, J.M. Muños-Escolano, J. Otal, N.N. Semko, Locally nilpotent linear groups with restrictions on their subgroups of infinite central dimension, Geometriae Dedicata, 138(1), 2009, pp. 69-81.

[10] J.M. Muños-Escolano, J. Otal, N.N. Semko, Periodic linear groups with the weak chain conditions on subgroups of infinite central dimension, Commun. Algebra, 36(2), 2008, pp. 749-763.

[11] A. Mann, Groups with dense normal subgroups, Israel J. Math., 6(1), 1968, pp. 1325.

[12] S.N. Chernikov, Groups with a dense system of complemented subgroups, Some Questions in Group Theory (in Russian), Ins. Mat. Akad. Nauk Ukr. SSR, Kiev, 1975, pp. 5-29.

[13] L.A. Kurdachenko, N.F. Kuzennyi, V.V. Pylaev, Infinite groups with a generalized dense system of subnormal subgroups, Ukr. Math. J., 33(3), 1981, pp. 313-316.

[14] L.A. Kurdachenko, V.E. Goretskii, Groups with a dense system of almost normal subgroups, Ukr. Math. J., 35(1), 1983, pp. 37-40.

[15] L.A. Kurdachenko, M.F. Kuzennyi, M.M. Semko, Groups with dense systems of infinite subgroups, Dokl. Akad. Nauk Ukr. SSR, Ser. A., 1985, pp. 7-9.

[16] L.A. Kurdachenko, N.F. Kuzennyi, N.N. Semko, Groups with a dense system of infinite almost normal subgroups, Ukr. Math. J., 43(7-8), 1992, pp. 904-908.

[17] N.N. Semko, On the construction of CDN[]-groups with elementary commutant of rank two, Ukr. Math. J., 49(10), 1997, pp. 1570-1577.

[18] N.N. Semko, On the structure of CDN[]-groups, Ukr. Math. J., 50(9), 1998, pp. 1431-1441.

[19] N.N. Semko, Structure of locally graded CDN( ]-groups, Ukr. Math. J., 50(11), 1998, pp. 1750-1754.

[20] N.N. Semko, Structure of locally graded CDN[ )-groups, Ukr. Math. J., 51(3), 1999, pp. $427-433$.

[21] M.R. Dixon, L.A. Kurdachenko, I.Ya. Subbotin, Ranks of groups: the tools, characteristics, and restrictions, New Jersey, 2017.

[22] Ya. Berkovich, Groups of prime power order, Vol. 1., Berlin, 2008.

[23] D. Gorenstein, Finite groups, New York, 1980. 
[24] L. Fuchs, Infinite abelian groups, Vol. 1., New York, 1970.

[25] G. Karpilovsky, Field theory, New York, 1988.

[26] M.R. Dixon, Sylow theory, formations and Fitting classes in locally finite groups, Singapore, 1994.

[27] B.A.F. Wehrfritz, Infinite linear groups, Berlin, 1973.

[28] M.I. Kargapolov, Yu.I. Merzlyakov, Foundations of group theory, Moskow, 1982.

[29] L.A. Kurdachenko, J. Otal, I.Ya. Subbotin, Groups with prescribed quotient groups and associated module theory, New Jersey, 2002.

\section{CONTACT INFORMATION}

Nikolaj N. Semko, Department of Mathematics, University of

Lilia V. Skaskiv, State Fiscal Service of Ukraine, Universytetska

Oksana street 31, Irpin, Kyiv region, Ukraine

A. Yarovaya E-Mail(s): dr.mykola.semko@gmail.com, lila_yonyk@ua.fm, yarovaoa@ukr.net

Received by the editors: 13.01.2019. 